\title{
Contingent color aftereffects: Reassessing old conclusions
}

\author{
LORRAINE G. ALLAN and SHEPARD SIEGEL \\ McMaster University, Hamilton, Ontario, Canada
}

\begin{abstract}
Although there is considerable evidence supporting an associative interpretation of contingent color aftereffects, there are data that appear inconsistent with this interpretation. New findings from seven experiments are presented indicating that, contrary to earlier claims, contingent color aftereffects are observed after induction with (1) single orthogonal black bars on colored backgrounds, (2) geometric forms, and (3) two orthogonal grids of the same color. The results of these experiments are relevant to an associative interpretation of contingent color aftereffects, as well as to assessing alternative interpretations of the phenomenon.
\end{abstract}

McCollough (1965) demonstrated a contingent color aftereffect (CCAE) by pairing complementary colors with orthogonally orientated grids. McCollough's orientation CCAE is readily induced. For example, during induction of the orientation CCAE, a grid constructed of black-and-green horizontal bars is alternated every few seconds with a grid constructed of black-and-magenta vertical bars. Following such induction, complementary color aftereffects contingent on grid orientation are noted -black-and-white assessment grids appear colored. In this example, the white space between black horizontal bars appears pinkish and the white space between black vertical bars appears greenish.

Since McCollough's (1965) demonstration of the orientation CCAE, many CCAEs have been described (see Siegel \& Allan, 1992). For example, there have been reports of CCAEs after pairing color with spatial frequency (e.g., Breitmeyer \& Cooper, 1972; Leppmann, 1973; Lovegrove \& Over, 1972; Stromeyer, 1972), movement direction (e.g., Hepler, 1968; Stromeyer \& Mansfield, 1970), dot size (e.g., MacKay \& MacKay, 1975), lightness of a frame surround (e.g., Siegel, Allan, \& Eissenberg, 1992), geometric form (e.g., Broerse \& Grimbeek, 1994; Humphrey, Herbert, Symons, \& Kara, 1994; Siegel, Allan, \& Eissenberg, 1992, 1994), and text (e.g., Allan, Siegel,

This research was supported by grants to L.G.A. and S.S. from the Natural Sciences and Engineering Research Council of Canada and the U. S. National Institute of Mental Health. Portions of the research were presented at the 1994 meeting of the Canadian Society for Brain, Behaviour, and Cognitive Science, Vancouver, BC, Canada, and at the 1995 meeting of the Eastern Psychological Association, Boston. The data in Experiments 4 and 5 are reported in a McMaster Honors BSc thesis submitted by V. Mavrou, and some of the data in Experiment 7 are reported in a McMaster Honors BSc thesis submitted by T. A. Rector. The authors gratefully acknowledge J. Gibbon for suggesting manipulations incorporated in Experiment 2, and T. Eissenberg and D. Mitchell for assistance in data collection. Correspondence should be addressed to L. G. Allan or S. Siegel, Department of Psychology, McMaster University, Hamilton, ON, Canada L8S 4K1 (e-mail: allan@mcmaster.ca or siegel@mcmaster.ca).
Collins, \& MacQueen, 1989; Humphrey, Skowbo, Symons, Herbert, \& Grant, 1994).

Several researchers have proposed that the CCAE is a manifestation of an association between the induction pattern and the induction color (see Siegel \& Allan, 1992). One of the earliest associative accounts of the orientation CCAE was provided by Murch (1976, p. 615): "the lined grid in inspection functions as a conditioned stimulus (CS) while color functions as the unconditioned stimulus (UCS). As a result of the pairing of the CS (lined grid) with the UCS (color) a conditioned response (CR) develops so that the adaptive response of the visual system to the color is evoked by the lined grid." According to an associative account, the CCAE represents a CR elicited by the color-paired cue - a grid of a certain orientation in McCollough's (1965) original demonstration. For example, for green-horizontal and magenta-vertical induction, one association is established between horizontal bars and green, resulting in a pink aftereffect on an achromatic horizontal grid; another association is established between vertical bars and magenta, resulting in a green aftereffect on an achromatic vertical grid. There is now considerable evidence supporting an associative interpretation of the CCAE (see Siegel \& Allan, 1992). There are data, however, that appear to be inconsistent with this interpretation. These data come from experiments evaluating CCAEs following induction with single bars, with geometric forms, and with only one color.

\section{Single-Bar Induction}

Foreit and Ambler (1978), in one of their experiments, attempted to induce an orientation CCAE with orthogonally oriented single bars (single-bar induction) rather than with orthogonally oriented grids. They compared two single-bar induction conditions. In one condition, the single bars were colored (e.g., a green horizontal bar and a magenta vertical bar) and the remainder of the screen was black. In the other condition, the single bars were black and the remainder of the screen was colored (e.g., a green background for the horizontal bar and a magenta back- 
ground for the vertical bar). Foreit and Ambler reported that orientation CCAEs were induced with the colored bars. After induction with a green horizontal bar and a magenta vertical bar, for example, a single achromatic horizontal bar appeared pink and a single achromatic vertical bar appeared green. However, orientation CCAEs were not induced with the black bars. After induction with a horizontal black bar on a green background and a black vertical bar on a magenta background, for example, the achromatic background surrounding a single black horizontal bar appeared achromatic, as did the achromatic background surrounding a single black vertical bar. Since orientation (CS) was paired with color (UCS) in both single-bar induction conditions, it would be expected, on the basis of an associative account, that orientation CCAEs should be induced whether the single bars are colored or black.

\section{Geometric Form Induction}

In another experiment, Foreit and Ambler (1978) attempted to induce CCAEs with geometric forms (form induction) constructed from different arrangements of the same colored bars. They alternated two outline geometric forms presented in complementary colors (e.g., a green cross and a magenta square). They reported that CCAEs were not observed after induction with these geometric forms, and concluded that "line configurations or forms per se are difficult to link with color to produce McCollough effects [CCAEs]" (p. 302). More recently, a number of investigators have reported that CCAEs are observed after induction with simple geometric forms similar to those used by Foreit and Ambler (e.g., Broerse \& Grimbeek, 1994; Humphrey, Herbert, et al., 1994; Siegel et al., 1992, 1994). There is disagreement, however, among these investigators regarding the nature of these aftereffects. Siegel et al. $(1992,1994)$ suggested that the induced aftereffects are spatiotopic; that is, that the aftereffects are contingent on form, and forms (like other patterns) can serve as CSs eliciting chromatic CRs that are CCAEs. Broerse and Grimbeek (1994) and Humphrey, Herbert, et al. (1994), in contrast, suggested that the induced aftereffects are retinotopic, and that forms constructed of the same orientation components do not elicit spatiotopic CCAEs.

\section{Same-Color Induction}

Humphrey, Dodwell, and Emerson (1985) attempted to induce orientation CCAEs with orthogonal grids of the same color (same-color induction) rather than of complementary colors. They reported that after same-color induction (e.g., green-horizontal and green-vertical), CCAEs were not induced on either grid orientation. According to an associative account, same-color induction should result in an aftereffect of the same color (pink, for the above example) on both orientations. For same-color induction, as for complementary-color induction, two associations should be established. During green-horizontal and green-vertical induction, for example, one associa- tion should be established between the horizontal orientation and the color green, resulting in a pink aftereffect on an achromatic horizontal grid; another association should be established between the vertical orientation and the color green, resulting in a pink aftereffect on an achromatic vertical grid.

The purpose of the present experiments was to evaluate further CCAEs induced with orthogonally oriented single bars, with geometric forms, and with same-color grids. The results of these experiments are relevant to an associative interpretation of CCAEs, as well as to current alternative interpretations of the phenomenon, especially the error-correction account developed by Dodwell and Humphrey (1990, 1993; Dodwell, 1992) and the perceptual learning account recently proposed by Bedford (1995).

\section{GENERAL METHOD}

\section{Subjects}

In all experiments except Experiment 6, the subjects were male and female students enrolled in introductory psychology at McMaster University who received course credit for their participation. These subjects had no previous experience in contingent aftereffect tasks. In Experiment 6, the subjects were male and female adults paid for their participation in the experiment. They either had no prior experience in contingent aftereffect tasks or had previously participated in a single contingent aftereffect experiment not less than 1 year prior to participation in the present experiment.

\section{Apparatus}

Temporal parameters, stimulus presentation, stimulus color, and recording of responses were controlled by a Macintosh (IIsi or LC475) computer equipped with an 8-bit video display card. Stimuli were displayed in the center of an Apple color monitor (Model M0401PA). RGB values, luminance values $\left(\mathrm{cd} / \mathrm{m}^{2}\right)$, and chromaticity values (CIE $x$ - and $y$-coordinates) were the same as those used in previous research (see Allan \& Siegel, 1991).

\section{Procedure}

An experimental session consisted of four phases: practice assessment, preinduction assessment, induction, and postinduction assessment. The room lights were off during the four phases, but were turned on for 2 min between induction and postinduction assessment to minimize the influence of simple afterimages. A variant of the method of constant stimuli (Allan, Siegel, Toppan, \& Lockhead, 1991) was used in the assessment phases. The experimenter was present in the room throughout the session, but did not observe the stimulus display.

Instructions were provided to the subject before each phase. Before each assessment phase, subjects were told (or reminded) that they were participating in a color discrimination task. Before the induction phase, subjects were told that they should observe the screen. Background music was played during induction. Unless otherwise specified (Experiment 4), subjects were not given any instructions regarding eye fixation, but were told (or reminded) before each phase to maintain their head in an upright position. A chinrest was not used.

\section{EXPERIMENT 1}

Orientation CCAEs are usually induced with orthogonally oriented and complementarily colored grids. Foreit and Ambler (1978) demonstrated that orientation CCAEs can also be induced with orthogonally oriented 
and complementarily colored single bars. Although Foreit and Ambler were able to demonstrate orientation CCAEs with colored bars on a black background, they were unsuccessful when the bars were black and the background was colored. In Experiment 1 we compared, as did Foreit and Ambler, CCAEs induced with orthogonally oriented and complementarily colored single bars on a black background with CCAEs induced with orthogonally oriented single black bars on complementarily colored backgrounds.

\section{Method \\ Stimuli \\ The induction and assessment stimuli were single bars presented at the center of the monitor screen. The bar was $5.9 \times .6 \mathrm{~cm}$, sub- tending approximately $4.8^{\circ} \times .5^{\circ}$ of visual angle.}

\section{Procedure}

Induction. Two orthogonally oriented bars alternated every $2 \mathrm{sec}$ during the 15 -min induction phase ( 225 presentations of each bar). There were two groups ( $n=10$ subjects per group). In the coloredbar group, single colored bars were presented on a black background: a single green horizontal bar and a single magenta vertical bar. The black background filled the monitor screen $(23.5 \times$ $17.5 \mathrm{~cm}$, subtending approximately $19.0^{\circ} \times 14.2^{\circ}$ of visual angle). In the black-bar group, single black bars were presented on a colored background: a single black horizontal bar on a green background and a single black vertical bar on a magenta background. The colored background filled the monitor screen.

Assessment. For both pre- and postinduction assessments, there were 50 presentations of a horizontal bar and 50 presentations of a vertical bar. For the colored-bar group, the background was black, and the bar could be one of five colors: one of two shades of pale pink ( $\mathrm{P} 2$ and $\mathrm{P} 1$, with $\mathrm{P} 2$ being more saturated than $\mathrm{P} 1$ ), one of two shades of pale green ( $G 2$ and $G 1$, with $G 2$ being more saturated than $\mathrm{G} 1$ ), or achromatic $(0)$. Bar orientation (horizontal or vertical) and color (P2, P1, 0, G1, G2) were randomly ordered, with the restriction that each bar orientation was presented in each color 10 times. On each of the 100 presentations, the subject indicated whether the bar appeared "green" or "pink." For the black-bar group, it was the background that was one of the five colors, and the subject indicated whether the background appeared "green" or "pink." The assessment stimulus remained on the screen until the subject responded. The next assessment stimulus was presented $1 \mathrm{sec}$ after the experimenter entered the subject's response on the computer keyboard.

Subjects were familiarized with the assessment procedure prior to the start of the experiment. Before preinduction assessment, subjects received 16 practice trials. The four unsaturated colors $(\mathrm{P} 2$, P1, G1, G2) were used. Each of the eight color-orientation combinations was presented twice (in random order). In contrast to the preand postinduction assessments, feedback was provided on these practice trials. Subjects were informed that their response was "correct" or "error" via the speech synthesis capability of the computer used to control the experiment.

\section{Results and Discussion}

The mean number of "green" responses $(\# \mathrm{G})$ to each bar orientation, across the five assessment colors, was determined for each subject in pre- and in postinduction assessment. The range of values for $\# \mathrm{G}$ is $0-10(0=$ never judged as green, $10=$ always judged as green). Figure 1 displays \#G, averaged over subjects, in pre- and postinduction as a function of assessment bar orientation. Fig-

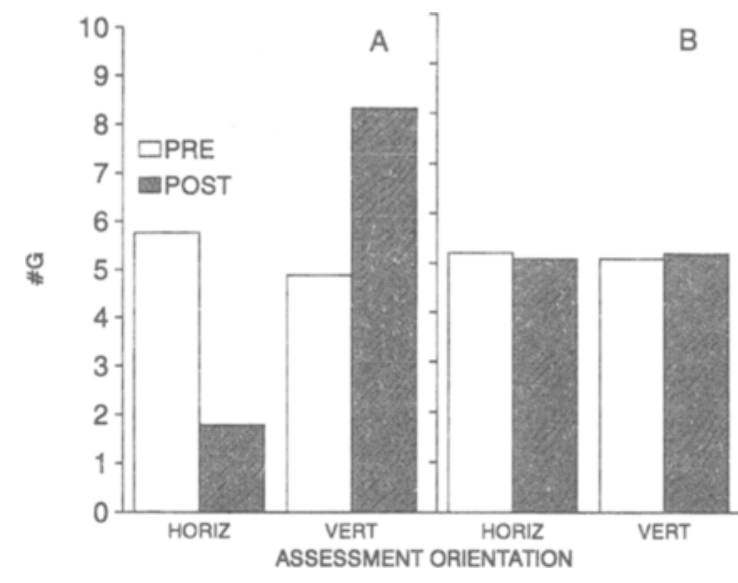

Figure 1. Mean pre-and postinduction \#G for each assessment bar orientation (horizontal and vertical) for the colored-bar induction group (A) and the black-bar induction group (B) in Experiment 1. \#G, mean number of "green" responses.

ure $1 \mathrm{~A}$ shows that, for the colored-bar group, \#G decreased from pre- to postinduction for the horizontal bar and increased for the vertical bar, indicating the induction of orientation CCAEs. Figure 1B shows that, for the black-bar group, there was little change in \#G from preto postinduction for both orientations, suggesting that orientation CCAEs were not induced.

A 2 (group: colored-bar or black-bar) $\times 2$ (assessment phase: pre or post) $\times 2$ (assessment orientation: horizontal or vertical) mixed-design analysis of variance (ANOVA) was performed on \#G to statistically evaluate the presence of orientation CCAEs after induction with colored bars and the absence of orientation CCAEs after induction with black bars. The three-way interaction between group, assessment phase, and assessment orientation was significant $[F(1,18)=36.48, p<.001]$. Planned comparisons ${ }^{1}$ revealed that the interaction between assessment phase and assessment orientation was significant for the colored-bar group $[F(1,18)=77.48, p<.001]$, confirming that orientation CCAEs were induced. Planned comparisons also revealed that the interaction between assessment phase and assessment orientation was not significant for the black-bar group $(F<1, p>.05)$, confirming that orientation CCAEs were not induced.

As described by Siegel et al. (1992), the shift in \#G from pre- to postinduction can be summarized by a single contingent aftereffect score. The aftereffect score compares the difference between $\# \mathrm{G}$ to the two orientations in postinduction to that difference in preinduction. In postinduction, \#G to the vertical orientation (induced in magenta) should be larger than \#G to the horizontal orientation (induced in green); in preinduction, \#G to the two grid orientations should be about the same. An aftereffect score was determined for each subject. On the basis of this score, all 10 subjects in the colored-bar group displayed orientation CCAEs, whereas only 5 of the 10 subjects in the black-bar group displayed orientation CCAEs. 
The results from this experiment are in agreement with those of Foreit and Ambler (1978). CCAEs can be induced with orthogonally oriented and complementarily colored single bars, but apparently not with orthogonally oriented single black bars on complementarily colored backgrounds.

\section{EXPERIMENT 2}

Foreit and Ambler (1978) noted that the ratio of black area to colored area was different in the two induction conditions; specifically, that the black-to-color ratio was greater in the colored-bar condition than in the black-bar condition. They suggested that increasing the black-tocolor ratio in black-bar induction might result in an orientation CCAE, but they did not investigate this possibility. In Experiment 2, the black-to-color ratio in black-bar induction was manipulated by varying both the size of the black bar and the size of the colored background.

\section{Method}

\section{Stimuli}

As in Experiment 1, the induction and assessment stimuli were single bars presented at the center of the monitor screen. The bar was black and was presented on a colored background. There were two bar sizes and two background sizes. The small bar, bar bas $_{\text {, w }}$ the same as that used in Experiment $1(5.9 \times .6 \mathrm{~cm})$. The large bar, $\operatorname{bar}_{L}$, had the same aspect ratio as bar $_{\mathrm{S}}$ but was twice the size $(11.8$ $\times 1.2 \mathrm{~cm}$ ). The large background, ground ${ }_{L}$, was $17.5-\mathrm{cm}$ square (the largest square possible on the Apple M0401PA color monitor), and the small background, ground $\mathrm{s}$, was $14.7-\mathrm{cm}$ square. For both ground $_{\mathfrak{L}}$ and ground $\mathrm{s}$, the screen around the colored background was black.

\section{Procedure}

Induction. As with the black-bar group in Experiment 1, two orthogonal black bars on colored backgrounds alternated every 2 sec during the 15 -min induction phase ( 225 presentations of each bar): a single black horizontal bar on a green background alternated with a single black vertical bar on a magenta background. There were four groups ( $n=11$ subjects per group), resulting from the two bar sizes being presented on the two background sizes: $\operatorname{bar}_{\mathrm{S}} / \operatorname{ground}_{\mathrm{L}}$,

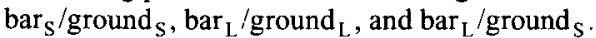

Assessment. Assessment was the same as for the black-bar group in Experiment 1. The size of the bar and the size of the background were the same in assessment as that used in induction.

\section{Results and Discussion}

Figure 2 displays mean pre- and postinduction \#G values for each of the four groups. Figure 2 indicates that, in general, \#G decreased from pre- to postinduction for the horizontal bar orientation and increased for the vertical bar orientation. The only exception was the horizontal bar orientation in the $\operatorname{bar}_{\mathrm{S}} /$ ground $_{\mathrm{L}}$ group, the group with the smallest black-to-color ratio (Figure 2A).

To evaluate the statistical significance of the interaction between assessment phase and assessment orientation for each of the four groups, a 4 (group) $\times 2$ (assessment phase) $\times 2$ (assessment orientation) mixed-design ANOVA was performed on $\# \mathrm{G}$. The three-way interaction between group, assessment phase, and assessment orientation $[F(3,40)=2.84, p<.05]$ was significant, indicating that
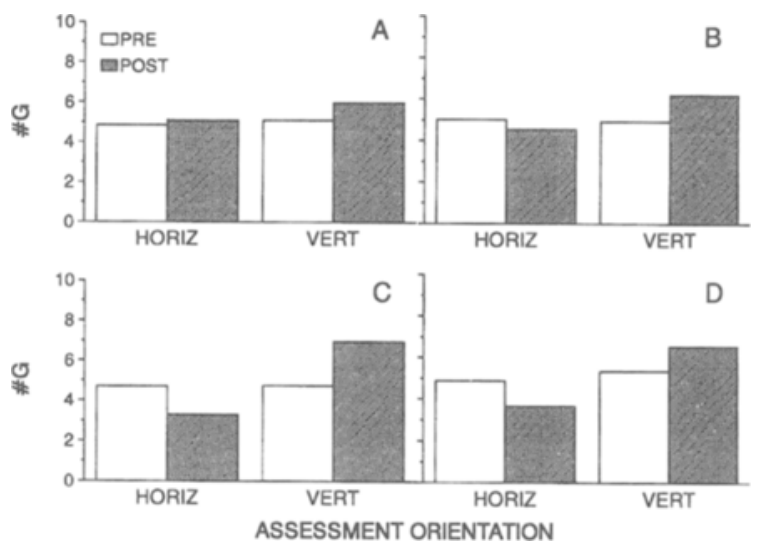

Figure 2. Mean pre- and postinduction \#G for each assessment bar orientation (horizontal and vertical) for the four induction groups in Experiment 2: (A) bar ${ }_{S}$ ground $_{L}$, (B) bar $_{S}$ /ground $_{S}$, (C) bar $_{L} /$ ground $_{L}$, and (D) bar $_{L} /$ ground $_{S}$. \#G, mean number of "green" responses.

the induction of reliable CCAEs was dependent on group. Planned comparisons revealed that the interaction between assessment phase and assessment orientation was significant for three of the groups [ bar $_{\mathrm{s}} /$ ground $_{\mathrm{s}}, F(1,40)=$ $5.56, p<.03$; bar $_{\mathrm{L}} /$ ground $_{\mathrm{L}}, F(1,40)=16.80, p<.001$; $\operatorname{bar}_{L} /$ ground $\left._{\mathrm{S}}, F(1,40)=10.67, p<.01\right]$, but was not significant for the bar $_{\mathrm{S}} /$ ground $_{\mathrm{L}}$ group $[F(1,40)<1]$.

As in Experiment 1, an aftereffect score was determined for each subject. Nine of the 11 subjects displayed orientation CCAEs in the bar ${ }_{\mathrm{S}}$ ground $_{\mathrm{S}}$ group, all 11 subjects did in the bar $_{L} /$ ground $_{L}$ group, and 10 did in the $\operatorname{bar}_{L} /$ ground $_{S}$ group. In contrast, only 4 subjects displayed an appropriate CCAE in the bar $_{S} /$ ground $_{\mathrm{L}}$ group.

The results from the present experiment clearly show that orthogonally oriented single black bars on complementarily colored backgrounds can be effective induction stimuli. As Foreit and Ambler (1978) suggested, black bar size relative to colored background size is an important factor. When the black bar was small relative to the colored background, as in Foreit and Ambler, as in Experiment 1 , and as in the bar $_{\mathrm{S}} /$ ground $_{\mathrm{L}}$ group of the present experiment, orientation CCAEs were not induced. Experiment 2 showed that when the relative size of the black bar to the colored background was increased, either by increasing the size of the bar or by decreasing the size of the background, orientation CCAEs were induced.

The results of Experiment 2 are compatible with an associative account of CCAEs. It is well established that the size of the CR depends on CS salience-the more salient the CS, the larger the CR (see Rescorla \& Wagner, 1972). Siegel and Allan (1985) have shown that the size of the orientation CCAE also depends on CS salience-the more salient the orientation CS, the larger the orientation CCAE. The results from Experiment 2 suggest that the influence of the black-to-color ratio on the size of the orientation CCAE is attributable to the salience of the orientation CS. A larger black-to-color ratio provides a more salient black orientation $\mathrm{CS}$ and results in a stronger orientation $\mathrm{CCAE},{ }^{2}$ 


\section{EXPERIMENT 3}

Foreit and Ambler (1978) found that, after induction with single colored bars, the orientation CCAE was elicited by grids as well as single bars. This result is expected on the basis of an associative account of CCAEs. According to an associative account, there should be CS generalization-CCAEs induced with one type of orientation stimulus should generalize to other stimulus patterns of the same orientation (see Allan \& Siegel, 1986; Skowbo, 1984). Experiment 3 was conducted to examine further the role of CS generalization. In Experiment 3, CCAEs were induced with grids and assessed with single bars.

\section{Method}

\section{Stimuli}

The induction stimuli were horizontal and vertical grids (approximately $5.9-\mathrm{cm}$ square, subtending approximately $4.8^{\circ}$ of visual angle) presented at the center of a black monitor screen. Each grid was composed of alternating black and nonblack bars of equal width ( 10 black bars alternating with 10 nonblack bars). The assessment stimuli were the single bars from Experiment $1(5.9 \times$ $.6 \mathrm{~cm}$ ). The single assessment bars were identical in size to the individual bars of the induction grid.

\section{Procedure}

Induction. A green horizontal grid alternated every $2 \mathrm{sec}$ with a magenta vertical grid during the 15 -min induction phase (225 presentations of each grid).

Assessment. There were two assessment groups $(n=10$ subjects per group). For the colored-bar group, assessment (practice, preinduction, postinduction) was the same as for the colored-bar group in Experiment 1: The subject categorized, as green or pink, the color of a bar that was presented on a black background. For the black rbar group, assessment was the same as for the black-bar group of Experiment 1 (except that the colored background was $8.8-\mathrm{cm}$ square): The subject categorized the color of the background that contained a black bar.

\section{Results and Discussion}

Figure 3 displays \#G, averaged over subjects, in preand postinduction as a function of assessment bar orientation. For the colored-bar group (Figure $3 \mathrm{~A}$ ), \#G decreased from pre- to postinduction for the horizontal bar and increased for the vertical bar. After induction with a green horizontal grid and a magenta vertical grid, a horizontal bar appeared more pink and a vertical bar appeared more green. The same pattern was seen for the black-bar group (Figure 3B). After induction with a green horizontal grid and a magenta vertical grid, the background surrounding a black horizontal bar appeared more pink and the background surrounding a black vertical bar appeared more green.

To evaluate the statistical significance of the interaction between assessment phase and assessment orientation shown in Figure 3, a 2 (assessment group) $\times 2$ (assessment phase) $\times 2$ (assessment orientation) mixed-design ANOVA was performed on $\# \mathrm{G}$. The two-way interaction between assessment phase and assessment orientation was significant $[F(1,18)=72.84, p<.001]$, indicating

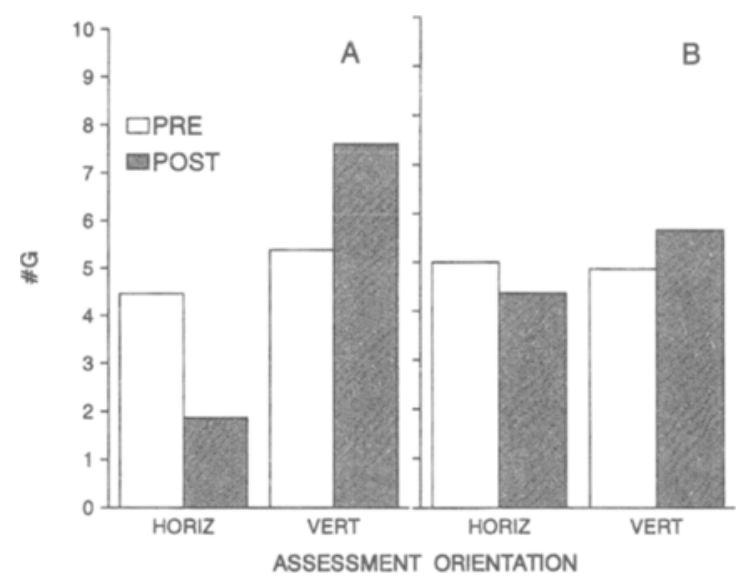

Figure 3. Mean pre- and postinduction \#G for each assessment bar orientation (horizontal and vertical) for the colored-bar assessment group (A) and the black-bar assessment group (B) in Experiment 3. \#G, mean number of "green" responses.

the presence of reliable orientation CCAEs. The three-way interaction between assessment group, assessment phase, and assessment orientation was also significant $[F(1,18)=$ $21.23, p<.001]$, indicating that the size of the CCAE depended on assessment group. Planned comparisons revealed that although the CCAE was smaller when assessed on a background than on a bar, both assessments showed significant CCAEs [black-bar, $F(1,18)=7.71, p<.02$; colored-bar, $F(1,18)=86.37, p<.001]$.

An aftereffect score was determined for each subject: All 10 subjects displayed CCAEs when assessed with a bar, and 8 did when assessed with a background.

As expected on the basis of an associative account, CCAEs induced with conventional stimuli (i.e., grids) transfer to single bars, whether the CCAE is assessed on the bar or on the background. The larger CCAE in the colored-bar group than in the black-bar group suggests a greater similarity between the orientation grid CS in induction and the orientation assessment $\mathrm{CS}$ in the coloredbar group than in the black-bar group.

\section{EXPERIMENT 4}

Several investigators (e.g., Dodwell \& Humphrey, 1990; Foreit \& Ambler, 1978; Skowbo, 1984) have suggested that, on the basis of an associative interpretation of CCAEs, it would be expected that a wide variety of stimuli, following pairing with color, should elicit CCAEs. Contrary to this prediction, Foreit and Ambler reported that alternate presentations of colored geometric forms, constructed from different arrangements of the same colored line segments, did not establish form CCAEs. In contrast, Siegel et al. (1992) reported that such simple geometric forms can elicit form CCAEs.

Broerse and Grimbeek (1994) and Humphrey, Herbert, et al. (1994) provided an account of the different findings 
reported by Foreit and Ambler (1978) and by Siegel et al. (1992). According to Broerse and Grimbeek and Humphrey et al., Foreit and Ambler instructed their subjects to scan the forms during induction. ${ }^{3}$ Siegel et al. gave their subjects no fixation instructions. Broerse and Grimbeek and Humphrey et al. hypothesized that, with no specific instructions to scan, Siegel et al.'s subjects fixated in the center of each form. Broerse and Grimbeek and Humphrey et al. suggested that Siegel et al.'s apparent demonstration of a form CCAE was, in fact, a demonstration of a retinotopic color aftereffect. That is, Siegel et al.'s results represent a retinally specific rather than a form-specific color aftereffect.

Broerse and Grimbeek (1994) and Humphrey, Herbert, et al. (1994) evaluated the hypothesis that the color aftereffect described by Siegel et al. (1992) was retinotopic by giving their subjects specific fixation instructions. Both Broerse and Grimbeek and Humphrey et al. demonstrated that, with specific fixation instructions, retinotopic color aftereffects can be obtained. They then inferred that Siegel et al. (1992) would not have obtained form CCAEs if their subjects had been instructed to scan the forms. Siegel et al. (1994) assessed this inference. They compared subjects who were given no explicit scanning instructions (like those in the Siegel et al., 1992, study) with subjects who were given explicit instructions to scan the forms. Both scanning and noninstructed groups displayed CCAEs, and there were no significant differences between the groups.

The results reported by Siegel et al. (1994) do not support the hypothesis advanced by Humphrey, Herbert, et al. (1994) and by Broerse and Grimbeek (1994) that instructions to scan would eliminate (or at least attenuate) the color aftereffect. Rather, Siegel et al.'s results suggest that scanning subjects $d o$ display form CCAEs. Experiment 4 provides additional evidence that nonfixating subjects display form CCAEs. In Experiment 4, subjects given explicit instructions to scan were compared with subjects given explicit instructions to fixate. To help the subject comply with the instructions, the scan subjects were instructed to follow a fixation point that traced the induction forms, and the fixate subjects were instructed to fixate a stationary point located in the middle of the induction forms.

\section{Method}

\section{Stimuli}

The induction and assessment stimuli were outline geometric forms, a square and a "tic-tac-toe" cross, used by Siegel et al. (1992, 1994) and Humphrey, Herbert, et al. (1994) and similar to the forms used by Foreit and Ambler (1978) and Broerse and Grimbeek (1994). Each form consisted of the same four line segments, $7.3 \times 0.6 \mathrm{~cm}$ (length $\times$ width), subtending approximately $6.0^{\circ} \times 0.5^{\circ}$ of visual angle (see Figure 4). The line segments were colored, and the form was presented at the center of a black monitor screen. The cross was constructed from the square by moving each side $2.5 \mathrm{~cm}$ toward the middle.

\section{Procedure}

Induction. The two colored forms alternated every $3 \mathrm{sec}$ during the 20 -min induction phase ( 200 presentations of each form). There
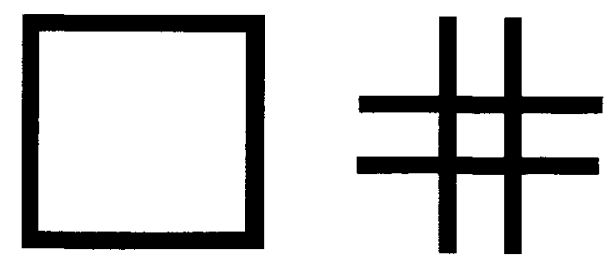

Figure 4. The geometric forms used in Experiments 4 and 5. Each form was constructed of four colored bars (shown as black in the figure).

were two induction groups ( $n=10$ subjects per group). Subjects in the fixate group were instructed to fixate on a small white square (2 pixels square) that appeared at the center of each form. Subjects in the scan group were instructed to follow the small square as it moved clockwise around the outside boundary of the form. The fixation square appeared in 12 locations and changed position every $.25 \mathrm{sec}$ as it progressed around the form.

Color-form pairing was counterbalanced. In each induction group, 5 subjects observed a green cross alternating with a magenta square, and the other 5 subjects observed a magenta cross alternating with a green square.

Assessment. Assessment was identical for the two groups of subjects. There was no fixation or moving point, and no explicit instructions regarding scanning were given to the subjects. For both preand postinduction assessments, there were 50 presentations of the square and 50 presentations of the cross. In all other respects, assessment was the same as for the color-bar group in Experiment 1that is, the subject categorized the color of the geometric form as green or pink.

\section{Results and Discussion}

Figure 5 displays \#G, averaged over subjects, in preand postinduction. For simplicity of presentation, the data in Figure 5 were collapsed across color-form pairing; the bars labeled "green" represent the assessment data for the form induced in green, and the bars labeled "magenta" represent the assessment data for the form induced in magenta. The results summarized in Figure 5 indicate that, for both instruction groups, \#G decreased from preto postinduction for the form presented in green during induction and increased for the form presented in magenta; the form that was green during induction appeared to be more pink and the form that was magenta during induction appeared to be more green.

To evaluate the statistical significance of the interaction between assessment phase and induction color shown in Figure 5 and to compare the size of the aftereffect between the two instruction groups, a 2 (instruction group) $\times 2$ (assessment phase) $\times 2$ (assessment form) mixed-design ANOVA was performed on \#G. The outcome of this analysis confirmed the trends shown in Figure 5. The interaction between assessment phase and induction color was significant $[F(1,18)=14.21, p<.01]$. The main effect of instruction group was not significant, nor did instruction group interact significantly with either of the other factors. On the basis of the aftereffect scores, 8 of the 10 subjects in each instruction group displayed a CCAE.

The results of this experiment indicate that CCAEs were induced in both instruction groups, and that the size 


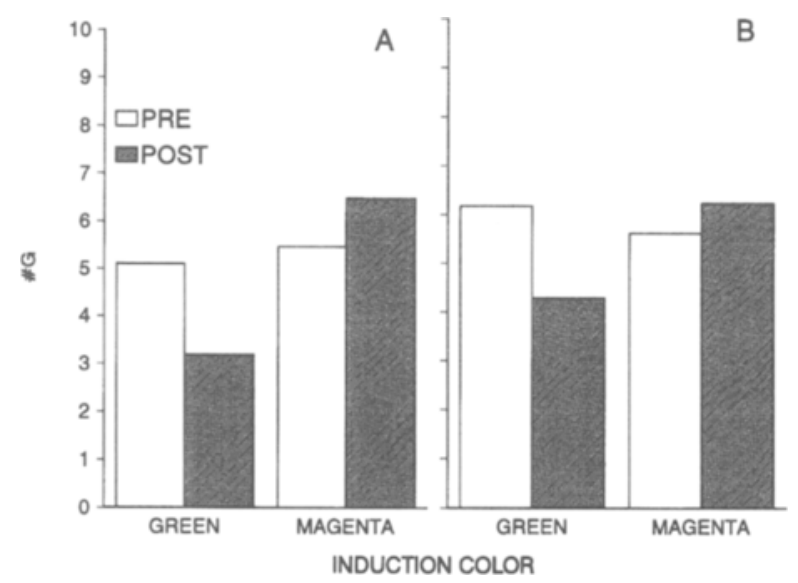

Figure 5. Mean pre- and postinduction $\#$ G for each induction color (green and magenta) for the scan induction group (A) and the fixate induction group (B) in Experiment 4. \#G, mean number of "green" responses.

of the CCAEs did not differ between the two instruction groups. These results confirm our earlier reports regarding form CCAEs (Siegel et al., 1992, 1994). Contrary to the claim of Broerse and Grimbeek (1994) and Humphrey, Herbert, et al. (1994), subjects displayed CCAEs after induction with geometric forms whether they were instructed to fixate the center of the form or instructed to scan the form by following a moving point. Moreover, the size of the CCAE did not differ between the two instruction groups. The results provide further evidence that spatiotopic features of visual forms, constructed by rearrangement of the same orientation components, can contingently elicit color aftereffects. As suggested by several investigators (e.g., Foreit \& Ambler, 1978; Siegel et al., 1992), such a finding is consistent with an associative interpretation of CCAEs.

\section{EXPERIMENT 5}

Although the subjects in the two groups in Experiment 4 were given different instructions, there is no independent evidence that they followed the instructions. It could be argued that the scan subjects ignored the instructions; that is, they did not follow the moving point, but rather fixated on the center of the induction form. According to this analysis, the two groups did not differ because both groups fixated, and thus the aftereffect seen in each group may have been retinotopic. In Experiment 5, rather than attempting to manipulate scanning of the form, we rotated the form during each induction presentation. CCAEs seen on stationary forms after such rotated-form induction could not be retinally specific.

\section{Method}

\section{Stimuli}

The forms, a square and the tic-tac-toe cross, were smaller than those used in Experiment 4. Each line segment was $4.7 \times 0.6 \mathrm{~cm}$ (length $\times$ width), subtending approximately $3.8^{\circ} \times 0.5^{\circ}$ of visual angle. The cross was constructed from the square by moving each side $1.2 \mathrm{~cm}$ toward the middle.

\section{Procedure}

Induction. The two forms alternated every $5 \mathrm{sec}$ during the 20min induction phase ( 120 presentations of each form). During each presentation, the form rotated clockwise through $180^{\circ}$. Color-form pairing was counterbalanced: 10 subjects observed a green rotating cross alternating with a magenta rotating square $(\mathrm{G}-\mathrm{Cr} / \mathrm{M}-\mathrm{Sq})$, and the other 10 subjects observed a green rotating square alternating with a magenta rotating cross $(\mathrm{G}-\mathrm{Sq} / \mathrm{M}-\mathrm{Cr})$.

Assessment. Pre- and postinduction assessment and practice were identical to those of Experiment 4-the assessment forms did not rotate.

\section{Results and Discussion}

Figure 6 displays \#G in pre- and postinduction as a function of induction form. The results summarized in Figure 6 indicate that, for both groups, \#G decreased from pre- to postinduction for the form presented in green in induction and increased for the form presented in magenta. A 2 (assessment phase) $\times 2$ (assessment form) repeated measures ANOVA was performed on $\# \mathrm{G}$, separately for each counterbalanced group. The interaction between assessment phase and assessment form was significant for both groups $[F \mathrm{~s}(1,9)>25, p s<.001]$. On the basis of aftereffect scores, each of the 20 subjects displayed a CCAE.

The results from this experiment demonstrate that CCAEs can be induced by rotating geometric forms. The induced CCAEs are not contingent on movement direction. Both induction forms rotated in the same direction. Also, the assessment forms did not rotate.

The results from Experiments 4 and 5, as well as earlier data (Broerse \& Grimbeek, 1994; Humphrey, Herbert, et al., 1994; Siegel et al., 1992, 1994), indicate that color aftereffects are readily induced with geometric forms under a variety of conditions. It appears that the failure reported by Foreit and Ambler (1978) is anomalous.

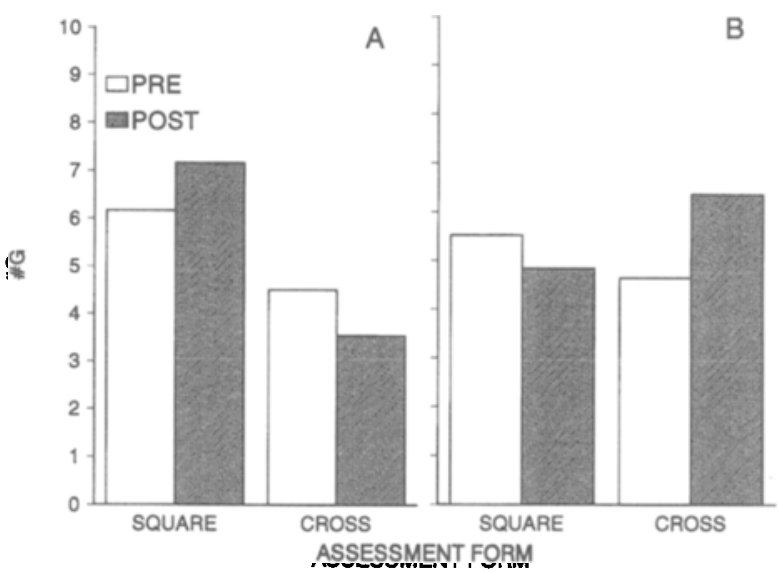

Figure 6. Mean pre- and postinduction \#G for each assessment form (square and cross) for the group that observed a green rotating cross alternating with a magenta rotating square $(A)$ and for the group that observed a green rotating square alternating with a magenta rotating cross (B) in Experiment 5. \#G, mean number of "green" responses. 
The results of Experiments 4 and 5 are not consistent with Broerse and Grimbeek's (1994) and Humphrey, Herbert, et al.'s (1994) suggestion that CCAEs would not be induced in nonfixating subjects. In Experiment 4, scanning was encouraged by elaboration of the display of colored forms with a fixation point that traced the shapes of the forms. In Experiment 5, the form was physically rotated. In both experiments, CCAEs were obtained, and the magnitude of the CCAE was unaltered by the scanning manipulations. Simple forms, constructed by rearrangements of the same components, can elicit spatiotopic CCAEs.

Siegel et al. (1992) and Foreit and Ambler (1978) argued that such form CCAEs would be supportive of an associative account. Thus, the results of Experiments 4 and 5 are not only contrary to suggestions that form CCAEs are simply retinotopic, but are also consistent with other findings suggesting that CCAEs are a manifestation of an association between the pattern and color used during induction (see Siegel \& Allan, 1992).

\section{EXPERIMENT 6}

Typically, CCAEs are induced by alternate presentations of complementarily colored patterns. Humphrey et al. (1985) attempted to induce orientation CCAEs by alternating presentations of same-color patterns (e.g., green-horizontal and green-vertical). They reported orientation CCAEs after complementarily colored induction but not after same-color induction. They concluded that for CCAE generation "it is necessary that two orthogonal inducing patterns not be viewed in light of the same color" (p. 343).

On the basis of an associative account, there is no special reason why the two orthogonal induction patterns must be presented in complementary colors. Experiment 6 was designed to investigate further the presence of orientation CCAEs after same-color induction. In an attempt to enhance the establishment of the CCAEs, the induction time was increased from the 10 min used in Humphrey et al. (1985) to $15 \mathrm{~min}$. As in the previous five experiments in this report, the CCAEs were assessed using the method of constant stimuli. The method of constant stimuli has been shown to be more sensitive in revealing the presence of CCAEs than the color-naming procedure used by Humphrey et al. (1985; see Allan et al., 1991; Humphrey, Herbert, et al., 1994; Humphrey, Skowbo, et al., 1994).

The assessment figures used by Humphrey et al. (1985) were composites consisting of various arrangements of the induction (and also noninduction) patterns. Such composite assessment figures allow for simultaneous color contrast during assessment (see Stromeyer, 1984). In fact, Humphrey et al. (1985) evaluated the role of simultaneous color contrast during assessment, and concluded that "in testing, the probability of obtaining a CAE [CCAE] report is greatly increased by presenting the subject with a test pattern designed to elicit two CAEs [CCAEs] of different hues" (p. 343). The assessment figures in Exper- iment 6 (as well as the previous experiments in this report) consist of only one pattern (i.e., one orientation). These assessment figures are not subject to simultaneous color contrast, and therefore are not biased in favor of revealing orientation CCAEs after complementarily colored induction relative to after same-color induction (see Allan \& Siegel, 1991, for a discussion of the role of simultaneous and successive color contrast with the method of constant stimuli).

\section{Method}

\section{Stimuli}

The induction and assessment stimuli were the square grids (horizontal and vertical) used in Experiment 3.

\section{Procedure}

Induction. There were three induction groups $(n=5$ subjects per group). Two groups received same-color induction; either both grids were green or both grids were magenta. A third group was induced with complementarily colored grids (green-horizontal and magenta-vertical). For all induction groups, the two grids alternated every $2 \mathrm{sec}$ during the $15-\mathrm{min}$ induction phase $(225$ presentations of each grid).

Assessment. For both pre- and postinduction assessment, all groups received 50 presentations of a horizontal grid and 50 presentations of a vertical grid. In all other respects, assessment was the same as in Experiment 1.

\section{Results and Discussion}

Figure 7 displays \#G, averaged over subjects, in preand postinduction as a function of assessment grid orientation. Figure 7A shows that after induction with a green horizontal grid and a magenta vertical grid, \#G decreased from pre- to postinduction for the horizontal orientation and increased for the vertical orientation, indicating the induction of orientation CCAEs. A 2 (assessment phase) $\times 2$ (assessment orientation) repeated measures ANOVA, conducted on $\# \mathrm{G}$, revealed that the interaction between

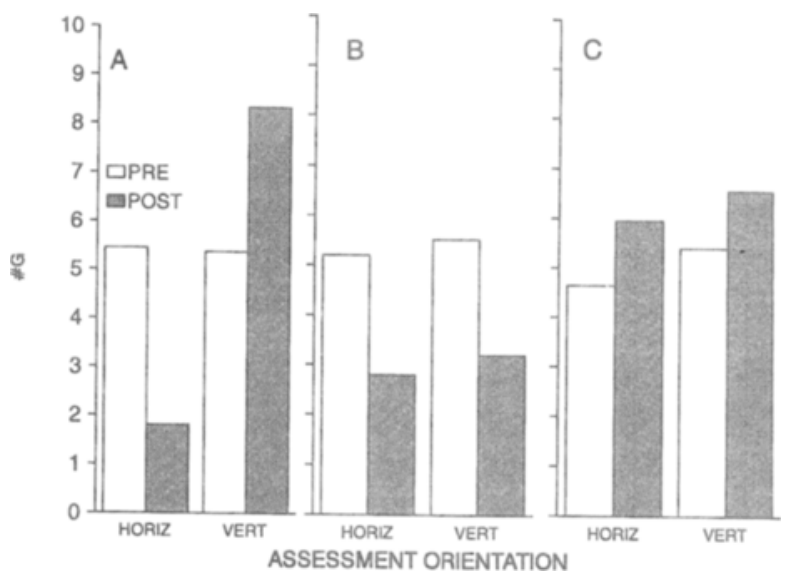

Figure 7. Mean pre- and postinduction \#G for each assessment grid orientation (horizontal and vertical) for the three induction groups in Experiment 6: (A) green-horizontal and magentavertical, $(B)$ green-horizontal and green-vertical, and $(C)$ magentahorizontal and magenta-vertical. \#G, mean number of "green" responses. 
assessment phase and assessment orientation shown in Figure $7 \mathrm{~A}$ was significant $[F(1,4)=17.07, p<.02]$; horizontal grids appeared to be pink and vertical grids appeared to be green.

Figure 7B shows that after same-color induction with green grids, \#G decreased from pre- to postinduction for both orientations, suggesting that both orientations appeared to be more pink. Figure $7 \mathrm{C}$ shows that after same-color induction with magenta grids, \#G increased from pre- to postinduction for both orientations, suggesting that both orientations appeared to be more green. A 2 (induction group: green or magenta) $\times 2$ (assessment phase) $\times 2$ (assessment orientation) mixed-design ANOVA, conducted on $\# \mathrm{G}$, revealed that the interaction between group and assessment phase shown in Figures 7B and 7C was significant $[F(1,8)=10.60, p<.02]$. After induction with green grids, both grid orientations appeared to be pink, and after induction with magenta grids, both orientations appeared to be green. These results are consistent with an associative account of CCAEs. During induction, both orientations CSs were paired with the same color, and both CSs elicited orientation CCAEs of the same color.

Analyses were conducted to determine whether the orientation CCAE induced with same-color grids differed in size from that induced with complementary-colored grids. One analysis compared the change in $\# \mathrm{G}$ to the horizontal orientation from pre- to postinduction for the two groups that were induced with green horizontal grids (complementarily colored induction and same-color induction with green). A 2 (induction group) $\times 2$ (assessment phase) mixed-design ANOVA revealed a significant main effect of assessment phase $[F(1,8)=20.48, p<.01]$. Neither the main effect of group nor the interaction of group with assessment phase was significant $\left[F_{\mathrm{s}}(1,8)<1\right]$. Another 2 (induction group) $\times 2$ (assessment phase) mixeddesign ANOVA compared the change in \#G to the vertical orientation from pre- to postinduction for the two groups that were induced with magenta vertical grids (complementarily colored induction and same-color induction with magenta). Again, the main effect of assessment phase was significant $[F(1,8)=11.41, p<.01]$, and again neither the main effect of group nor the interaction was significant $[F(1,8)=1.08, p>.05$ and $F(1,8)=2.18, p>.05$, respectively]. These analyses indicate that the orientation CCAEs induced with complementarily colored grids and with same-color grids did not differ significantly.

Experiment 6 was designed to enhance the establishment of orientation CCAEs by increasing the induction time, to improve the measurement of the CCAE by using the method of constant stimuli, and to remove biases in favor of revealing CCAEs after complementarily colored induction relative to revealing CCAEs after same-color induction. It is likely that both the longer induction and the more sensitive assessment were responsible for the observation of orientation CCAEs after same-color induction, and that the absence of biases resulted in orientation CCAEs after same-color induction that did not differ in size from those after complementarily colored induction.

\section{EXPERIMENT 7}

One purpose of Experiment 7 was to assess the reliability of same-color induction. A second purpose was to show that the aftereffect induced after same-color induction is, in fact, contingent on the orientations presented during induction. During same-color induction, two orientations are paired with the same color and both orientations should elicit orientation CCAEs. On the basis of an associative account, one would expect that if only one orientation (i.e., a nonalternating grid) were paired with color during induction, only the induced orientation would elicit an orientation $\mathrm{CCAE}$ - that is, only the orientation CS presented in induction would elicit the CCAE. ${ }^{4}$

Two induction procedures were compared in Experiment 7 . One group of subjects was induced with samecolor orthogonal grids. Another group was induced with one grid that was continuously present during the induction phase.

\section{Method}

\section{Stimuli}

As in Experiment 6, horizontal $(\mathrm{H})$ and vertical $(\mathrm{V})$ grids were used.

\section{Procedure}

Induction. The induction grids were green. For the HV group $(n=12)$, a green horizontal grid alternated every $2 \mathrm{sec}$ with a green vertical grid during the 15 -min induction phase ( 225 presentations of each grid). For the H-only group $(n=10)$, a green horizontal grid was continuously present throughout the 15 -min induction phase.

Assessment. Assessment was identical to that in Experiment 6.

\section{Results and Discussion}

Figure 8 displays the mean \#G in pre- and postinduction for the HV (Figure 8A) and H-only (Figure 8B) groups. Figure $8 \mathrm{~A}$ shows that, for group $\mathrm{HV}$, \#G decreased from pre- to postinduction for both orientations. After induction with a green horizontal grid alternating with a green vertical grid, both grid orientations appeared to be more

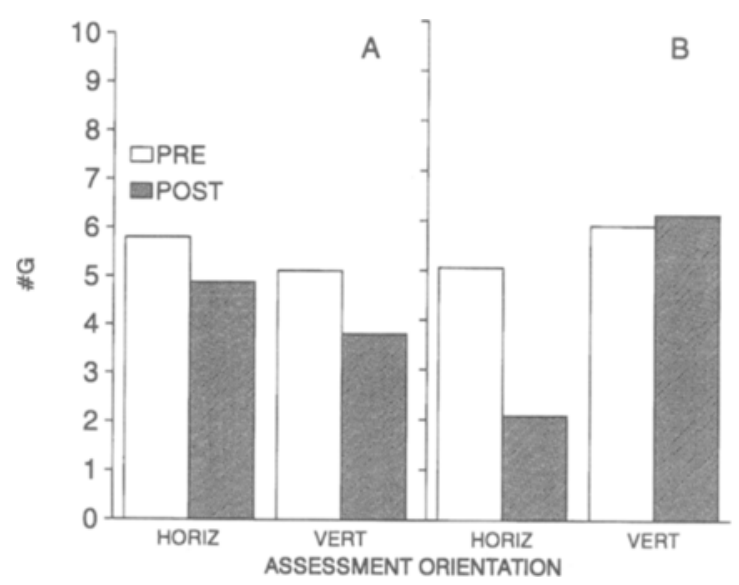

Figure 8. Mean pre- and postinduction \#G for each assessment grid orientation (horizontal and vertical) for induction groups horizontal-vertical (A) and horizontal-only (B) in Experiment 7. \#G, mean number of "green" responses. 
pink. Figure $8 \mathrm{~B}$ shows that, for the $\mathrm{H}$-only group, \#G decreased from pre- to postinduction only for the horizontal grid. After induction with a nonalternating green horizontal grid, only the horizontal grid appeared to be more pink.

To determine whether the difference between the two groups shown in Figure 8 was significant, a 2 (induction group) $\times 2$ (assessment phase) $\times 2$ (assessment orientation) mixed-design ANOVA was conducted on \#G. The significant three-way interaction $[F(1,20)=12.00, p<$ $.01]$ confirms that the two induction procedures had a differential effect on the two groups.

A 2 (assessment phase) $\times 2$ (assessment orientation) repeated measures ANOVA on the HV group data revealed that only the main effect of assessment phase was significant $[F(1,11)=32.11, p<.001]$. Planned comparisons showed that the decrease in \#G was significant for both orientations $[F(1,11)=10.71, p<.01$ for horizontal; $F(1,11)=35.52, p<.001$ for vertical], indicating that both orientations appeared to be more pink after induction. A similar ANOVA on the H-only group data revealed a significant interaction between assessment phase and assessment orientation $[F(1,9)=8.89, p<.02]$. Planned comparisons showed that the decrease in $\# \mathrm{G}$ was significant for the horizontal orientation $[F(1,9)=75.36$, $p<.001]$, but not for the vertical orientation $[F(1,9)<1]$, indicating that only the horizontal orientation appeared to be more pink after induction.

The results of Experiment 7 are consistent with an associative account of CCAEs. When two CSs (horizontal and vertical) are each paired with the same color (green), each elicits orientation CCAEs of the same color (pink). When only one CS (horizontal) is paired with color (green), only that CS elicits an orientation CCAE (pink).

\section{GENERAL DISCUSSION}

The seven experiments described in this report provide new data about CCAEs induced with orthogonally oriented single bars, with geometric forms, and with samecolor grids. The results of these experiments are relevant to an associative interpretation of CCAEs, as well as to current alternative interpretations of the phenomenon.

The data from Experiment 1 are consistent with those reported by Foreit and Ambler (1978). In Experiment 1, the induction patterns were orthogonally oriented single bars. Induction with complementarily colored bars on a black background resulted in orientation CCAEs, whereas induction with black bars on complementarily colored backgrounds did not. However, the results of Experiment 2 showed that orientation CCAEs can be induced with black bars on complementarily colored backgrounds when the black-to-color ratio is increased either by increasing the size of the single black bar or by decreasing the size of the colored background. The results of Experiment $3 \mathrm{dem}-$ onstrated that orientation CCAEs induced with grids generalize to single bars on black backgrounds and to backgrounds of single black bars.
Results of Experiments 4 and 5 are in agreement with other reports in the literature (e.g., Broerse \& Grimbeek, 1994; Humphrey, Herbert, et al., 1994; Siegel et al., 1992, 1994) that CCAEs can be induced with geometric forms. Experiments 4 and 5 also provide evidence against the hypothesis advanced by Broerse and Grimbeek and Humphrey, Herbert, et al. that scanning subjects would not display CCAEs after induction with geometric forms. The results of Experiment 4 showed that CCAEs were displayed both by subjects instructed to scan a moving square during induction and by subjects instructed to fixate on a stationary square during induction. Moreover, the size of the CCAE did not differ under the two induction instructions. Experiment 5 was designed to attenuate the effects of fixation on form CCAEs by rotating the forms during induction. Despite such rotation, form CCAEs were obtained.

The results of Experiments 6 and 7 indicated, contrary to an earlier report (Humphrey et al., 1985), that samecolor induction results in orientation CCAEs. After induction with same-color orthogonally oriented grids, both induction orientations appear to be colored, the color being the complement of the induction color. The samecolor induction and assessment procedures in Experiments 6 and 7 differed from those used by Humphrey et al. (1985). For example, the induction phase was longer and a more sensitive assessment procedure was used. In addition, the assessment figures were not biased, by simultaneous color contrast, in favor of revealing CCAEs after complementarily colored induction relative to same-color induction.

The results of the seven experiments are consistent with an associative interpretation of CCAEs. According to an associative interpretation, the CCAE represents a CR elicited by the color-paired cue. In Experiments 4 and 5 , the color-paired cue that elicited the CCAE was geometric form; in the other five experiments, the colorpaired cue was orientation (grid or single bar).

The results are also relevant to current alternative accounts of CCAEs - the error-correction account of Dodwell and Humphrey (1990, 1993; Dodwell, 1992) and the perceptual learning account recently proposed by Bedford (1995).

\section{Error-Correction Accounts of CCAEs}

A number of investigators have proposed accounts that view CCAEs as the result of recalibratory or errorcorrecting processes (e.g., Barlow, 1990; Barlow \& Foldiak, 1989; Dodwell, 1992; Dodwell \& Humphrey, 1990, 1993; Wolfe \& O'Connell, 1986). The various errorcorrection interpretations differ with respect to the conceptualization and function of the recalibratory process. The most detailed and developed of the error-correction accounts is the one proposed by Dodwell and Humphrey (1990, 1993; Dodwell, 1992).

According to Dodwell and Humphrey (1990, 1993; Dodwell, 1992), CCAEs are generated because the zero 
correlation that normally exists between color and pattern is violated by the high correlation imposed during induction. To maintain an internal representation of the zero correlation in the presence of the artificially high correlation during CCAE induction, the system recalibrates to decorrelate color and pattern. The recalibration is accomplished by shifting the adaptation level (Helson, 1964) or neutral point toward the overrepresented end of the color continuum. During induction with green-horizontal and magenta-vertical, for example, the neutral point for green in the presence of horizontals is shifted toward green and the neutral point for magenta in the presence of verticals is shifted toward magenta. In assessment, an achromatic horizontal grid will be on the magenta side of the neutral point and will appear pinkish, and an achromatic vertical grid will be on the green side of the neutral point and will appear greenish.

In the presentation of their error-correction account, Dodwell and Humphrey (1990, 1993; Dodwell, 1992) explicitly addressed form induction and same-color induction, but not single-bar induction.

Form induction. The error-correction account is equivocal on the outcome of form induction. Dodwell and Humphrey (1990) stated that there must be constraints on recalibration: "Contingent aftereffects do not appear every time we stare at a face or a building, or anything else that has a particular shape and color" (p. 82). They suggested that the stimuli that can contingently elicit color aftereffects belong to "a small class of rather special patterns" (p. 79). These special patterns, according to Dodwell's (1992) model of visual perception, are orthogonally oriented pairs such as horizontal and vertical grids, and wouId not include the forms that were demonstrably effective in the experiments described here. However, Dodwell and Humphrey (1990) subsequently and contrariwise stated that "we do not claim that all MEs [CCAEs] ... are contingent on particular patterns, or that all of them fall neatly into the separate vectorfield channels hypothesis that we favor. There could be many different reasons for setting up biases so that contingent aftereffects occur" (p. 87). Such a qualified version of the errorcorrection account could encompass the form induction data from Experiments 4 and 5.5

Same-color induction. The correlation between color and grid is undefined under same-color induction. In that sense, the normal zero correlation is not violated, there would be no recalibration, and orientation CCAEs would not be expected by the Dodwell and Humphrey (1990) error-correction account. Dodwell and Humphrey discussed the finding reported by Humphrey et al. (1985) that same-color induction did not elicit orientation CCAEs and concluded that complementary colors must be present in induction for reliable CCAEs to be induced. The data from Experiments 6 and 7 demonstrate that samecolor induction can result in orientation CCAEs. It is possible that such a finding may be incorporated by a revision of the error-correction model of CCAEs.

\section{Perceptual Learning Account of CCAEs}

According to Bedford's (1995) perceptual learning account, a CCAE will be induced when the two induction patterns are interpreted as referring to a single object and a real-world constraint about that object is violated. This is the case in the usual induction of orientation CCAEs involving two complementarily colored and orthogonally oriented grids. The two orthogonal grids can be interpreted as the same object tilted $90^{\circ}$ (or the same object viewed with the head tilted $90^{\circ}$ ). The constraint, that an object does not change color when the object or head is tilted, is violated during induction. This real-world constraint is preserved by an internal correction for the perceived color differences in such a way that different orientations of the same object will no longer have different colors. If the correction is complete during induction, magenta vertical bars and green horizontal bars should appear to be the same color (gray). This internal correction results in the orientation CCAE. When assessed with achromatic grids after induction, vertical bars will appear green and horizontal bars will appear pink.

Bedford (1995) cited the Foreit and Ambler (1978) single-bar data, the Foreit and Ambler geometric form data, and the Humphrey et al. (1985) same-color data, as supportive of her perceptual learning account of CCAEs.

Single-bar induction. With reference to the Foreit and Ambler (1978) single-bar experiment, Bedford (1995) suggested that during black-bar induction, "When the figure changed orientation it did not change color-it stayed black. Consequently, there is no discrepancy. Though the background changed color, it did not clearly change orientation" (p. 271). Since there was no violation of a realworld constraint, a CCAE should not be induced. The single-bar induction data from Experiment 2 demonstrate that induction with single black bars on colored backgrounds can result in orientation CCAEs.

Form induction. According to Bedford (1995), the square and cross stimuli used by Foreit and Ambler (1978) should be ineffective in inducing a CCAE "because the retinal image of a cross and the retinal image of a square cannot refer to the same object - assuming objects maintain rigidity. Consequently, pairing a red square and a green cross is not a discrepancy. Different objects can reflect different wavelengths of light. ... No internal malfunction will be detected, or corrected, and no illusory colors manifested" (pp. 264-265). Bedford did note that evidence for CCAEs, using forms like those in Foreit and Ambler, had been reported by Siegel et al. (1992). To encompass the Siegel et al. data, Bedford proposed that the research on object identity in apparent motion suggests that "even stimuli such as a square and a cross may be accepted as referring to the same object, though in nature they never can" (p. 269). She inferred that aftereffects contingent on such stimuli should be difficult to obtain. In support of this inference, she noted that aftereffects induced with geometric forms were not revealed when assessed with the color-matching procedure used by Foreit and Ambler, 
but were revealed when assessed with the more sensitive method of constant stimuli used by Siegel et al. The recent report by Broerse and Grimbeek (1994) indicates, however, that aftereffects induced with geometric forms are not difficult to obtain. Broerse and Grimbeek assessed the presence of the aftereffects with color naming, a relatively gross and insensitive measure.

The form induction data from Experiments 4 and 5, as well as earlier form induction data reported by Broerse and Grimbeek (1994), Humphrey, Herbert, et al. (1994), and Siegel et al. (1992, 1994), provide evidence that induction patterns that do not refer to the same object can reliably and readily elicit CCAEs.

Same-color induction. Under same-color induction, the grid object does not change color as its orientation changes, a real-world constraint would not be violated, and, according to Bedford (1995), orientation CCAEs should not be induced: "If the object does not appear to change color when the retinal orientation changes, then systems are working properly and there is no error to correct" (Bedford, 1995, p. 285). The data from Experiments 6 and 7 demonstrate that same-color induction does result in orientation CCAEs.

\section{Concluding Remarks}

In summary, the results of the present experiments are consistent with an associative account of CCAEs. We have indicated elsewhere (Allan \& Siegel, 1993) that error-correction analyses of CCAEs are, in fact, very similar to associative analyses. It is not surprising, then, that the results of the present experiments may be consistent with error-correction interpretations (e.g., Dodwell \& Humphrey, 1990), especially if caution is exercised with respect to specifying the exact stimuli necessary to engage the error-correction mechanism. The results reported here clearly are inconsistent with Bedford's (1995) perceptual learning account of CCAEs.

\section{REFERENCES}

Allan, L. G., \& Siegel, S. (1986). McCollough effects as conditioned responses: Reply to Skowbo. Psychological Bulletin, 100, 388-393.

Allan, L. G., \& Siegel, S. (1991). Characteristics of the indirect McCollough effect. Perception \& Psychophysics, 50, 249-257.

Allan, L. G., \& Siegel, S. (1993). McCollough effects as conditioned responses: Reply to Dodwell and Humphrey. Psychological Review, 100, 342-346.

Allan, L. G., Siegel, S., Collins, J. C., \& MacQueen, G. M. (1989). Color aftereffect contingent on text. Perception \& Psychophysics, 46, 105-113.

Allan, L. G., Siegel, S., Toppan, P., \& Lockhead, G. R. (1991). Assessment of the McCollough effect by a shift in psychometric function. Bulletin of the Psychonomic Society, 29, 21-24.

BARLOW, H. B. (1990). A theory about the functional role and synaptic mechanism of visual after-effects. In C. Blakemore (Ed.), Vision: Coding and efficiency (pp. 363-375). Cambridge: Cambridge University Press.

Barlow, H. B., \& FoldiaK, P. (1989). Adaptation and decorrelation in the cortex. In R. Durbin, C. Miall, \& G. Mitchison (Eds.), The computing neuron (pp. 54-72). New York: Addison-Wesley.

BEDFORD, F. L. (1995). Constraints on perceptual learning: Objects and dimensions. Cognition, 54, 253-297.
BreitMeyer, B. G., \& CoOper, L. A. (1972). Frequency-specific color adaptation in the human visual system. Perception \& Psychophysics, 11, 95-96.

Broerse, J., \& Grimbeek, P. (1994). Eye movements and the associative basis of contingent color aftereffects: A comment on Siegel, Allan, and Eissenberg (1992). Journal of Experimental Psychology: General, 123, 81-85.

DoDWELl, P. C. (1992). Perspectives and transformations. Canadian Journal of Psychology, 46, 510-538.

DoDWELl, P. C., \& HuMPHREY, G. K. (1990). A functional theory of the McCollough effect. Psychological Review, 97, 78-79.

DoDWELl, P. C., \& HumPhreY, G. K. (1993). What is important about McCollough effects? A reply to Allan and Siegel. Psychological Review, 100, 347-350.

Eissenberg, T., Allan, L. G., Siegel, S., \& Petrov, N. (1995). An associative interpretation of the indirect McCollough effect. Quarterly Journal of Experimental Psychology, 48B, 262-286.

FOREIT, K. G., \& AMBLER, B. A. (1978). Induction of the McCollough effect I: Figural variables. Perception \& Psychophysics, 24, 295-302.

Helson, H. (1964). Adaptation-level theory: An experimental and systematic approach to behavior. New York: Harper \& Row.

HEPLER, N. (1968). Color: A motion-contingent aftereffect. Science, 162, 376-377.

Humphrey, G. K., Dodwell, P. C., \& Emerson, V.F. (1985). The roles of pattern orthogonality and color contrast in the generation of patterncontingent color aftereffects. Perception \& Psychophysics, 38, 343-353.

Humphrey, G. K., Herbert, A. M., Symons, L. A., \& Kara, S. (1994). McCollough effect to "form": A local phenomenon. Journal of Experimental Psychology: General, 123, 86-90.

Humphrey, G. K., Skowbo, D., Symons, L. A., Herbert, A. M., \& GRANT, C. L. (1994). Text-contingent color aftereffects: A reexamination. Perception \& Psychophysics, 56, 405-413.

KePPEL, G. (1991). Design and analysis: A researcher's handbook. Englewood Cliffs, NJ: Prentice-Hall.

LEPPMANN, P. K. (1973). Spatial frequency dependent chromatic aftereffects. Nature, 242, 411-412.

Lovegrove, W. J., \& Over, R. (1972). Color adaptation of spatial frequency detectors in the human visual system. Science, 176, 541-543.

MACKAY, D. M., \& MaCKAY, V. (1975). Dichoptic induction of McCollough-type effects. Quarterly Journal of Experimental Psychology, 27, 225-233.

McCollovgr, C. (1965). Color adaptation of edge-detectors in the human visual system. Science, 149, 1115-1116.

Murch, G. M. (1976). Classical conditioning of the McCollough effect: Temporal parameters. Vision Research, 16, 615-619.

REsCORLA, R. A., \& WAGNER, A. R. (1972). A theory of Pavlovian conditioning: Variations in the effectiveness of reinforcement and nonreinforcement. In A. H. Black \& W. F. Prokasy (Eds.), Classical conditioning II: Current theory and research (pp. 64-99). New York: Appleton-Century-Crofts.

SiEgEL, S., \& Allan, L. G. (1985). Overshadowing and blocking of the orientation-contingent color aftereffect: Evidence for a conditioning mechanism. Learning \& Motivation, 16, 125-138.

Siegel, S., \& Allan, L. G. (1992). Pairings in learning and perception: Pavlovian conditioning and contingent aftereffects. In D. Medin (Ed.), The psychology of learning and motivation (Vol. 28, pp. 127160). New York: Academic Press.

Siegel, S., Allan, L. G., \& Eissenberg, T. (1992). The associative basis of contingent color aftereffects. Journal of Experimental Psychology: General, 121, 79-94.

Siegel, S., Allan, L. G., \& Eissenberg, T. (1994). Scanning and formcontingent color aftereffects. Journal of Experimental Psychology: General, 123, 91-94.

SkowBo, D. (1984). Are McCollough effects conditioned responses? Psychological Bulletin, 96, 215-226.

STROMEYER, C. F. (1972). Edge-contingent color after effects: Spatial frequency specificity. Vision Research, 12, 717-733.

STROMEYER, C. F. (1984). Orientation-specific color aftereffects and simultaneous color contrast. In L. Spillman \& B. R. Wooten (Eds.), Sensory experience, adaptation and perception (pp. 509-527). Hillsdale, NJ: Erlbaum. 
Stromeyer, C. F., \& Mansfield, R. J. W. (1970). Colored aftereffects produced with moving edges. Perception \& Psychophysics, 7, 108-114.

Wolfe J. M., \& O'Connell, K. M. (1986). Fatigue and structural change: Two consequences of visual pattern adaptation. Investigative Ophthalmology \& Visual Science, 27, 538-543.

\section{NOTES}

1. A two-way significant interaction between assessment phase (pre and post) and induction orientation indicates the induction of a reliable CCAE, whereas a nonsignificant interaction indicates that a CCAE was not induced. We followed the significant three-way interaction with groups with planned comparisons to explore which groups showed the two-way interaction-that is, the CCAE. Our use of planned comparisons in this experiment (and in other experiments reported in this manuscript) is consistent with the views expressed by Keppel (1991).

2. Although the putative CS (bar orientation) likely is more salient in the conditions favoring the expression of the bar-orientation CCAE, the putative UCS (background color) is likely less salient. That is, for each size display, a large black bar is surrounded by less colored area than is a smaller black bar. Thus, implicit in the associative interpretation of the results of Experiment 2 is that the CS manipulation is more effective than the UCS manipulation.

3. In fact, it is unclear whether or not Foreit and Ambler (1978) actually instructed their subjects to scan (see Siegel et al., 1994).

4. It should be noted that this procedure is quite different from that used in studies of the "indirect ME" (see Allan \& Siegel, 1991). During indirect ME induction, a chromatic grid (e.g., green horizontal) is alternated with another stimulus during induction (e.g., a gridless chromatic or achromatic square). An associative account of the indirect $\mathrm{ME}$ is presented in Eissenberg, Allan, Siegel, and Petrov (1995).

5. The arguments advanced by Humphrey, Herbert, et al. (1994) and Broerse and Grimbeek (1994) that CCAEs induced with geometric forms are retinotopic, rather than spatiotopic, do not derive from the errorcorrection account of CCAEs.

(Manuscript received August 29, 1995; revision accepted for publication April 2, 1996.) 